\title{
Single shot, double differential spectral measurements of inverse Compton scattering in the nonlinear regime
}

\author{
Y. Sakai, ${ }^{1}$ I. Gadjev, ${ }^{1}$ P. Hoang, ${ }^{1}$ N. Majernik, ${ }^{1}$ A. Nause, ${ }^{1}$ A. Fukasawa, ${ }^{1}$ O. Williams, ${ }^{1}$ \\ M. Fedurin, ${ }^{2}$ B. Malone ${ }^{2}$ C. Swinson, ${ }^{2}$ K. Kusche, ${ }^{2}$ M. Polyanskiy, ${ }^{2}$ M. Babzien, ${ }^{2}$ \\ M. Montemagno, ${ }^{2}$ Z. Zhong, ${ }^{2}$ P. Siddons, ${ }^{2}$ I. Pogorelsky, ${ }^{2}$ V. Yakimenko, ${ }^{2,5}$ T. Kumita, ${ }^{3}$ \\ Y. Kamiya, ${ }^{4}$ and J. B. Rosenzweig ${ }^{1}$ \\ ${ }^{1}$ UCLA Department of Physics and Astronomy, 405 Hilgard Avenue, Los Angeles, California 90095, USA \\ ${ }^{2}$ Brookhaven National Laboratory, Upton, New York 11973, USA \\ ${ }^{3}$ Tokyo Metropolitan University, Tokyo 192-0397, Japan \\ ${ }^{4}$ ICEPP, Tokyo University, Tokyo 113-0033, Japan \\ ${ }^{5}$ SLAC National Accelerator Laboratory, Menlo Park, California 94025, USA
}

(Received 2 January 2017; published 5 June 2017)

\begin{abstract}
Inverse Compton scattering (ICS) is a unique mechanism for producing fast pulses-picosecond and below-of bright photons, ranging from $\mathrm{x}$ to $\gamma$ rays. These nominally narrow spectral bandwidth electromagnetic radiation pulses are efficiently produced in the interaction between intense, well-focused electron and laser beams. The spectral characteristics of such sources are affected by many experimental parameters, with intense laser effects often dominant. A laser field capable of inducing relativistic oscillatory motion may give rise to harmonic generation and, importantly for the present work, nonlinear redshifting, both of which dilute the spectral brightness of the radiation. As the applications enabled by this source often depend sensitively on its spectra, it is critical to resolve the details of the wavelength and angular distribution obtained from ICS collisions. With this motivation, we present an experimental study that greatly improves on previous spectral measurement methods based on $\mathrm{x}$-ray $K$-edge filters, by implementing a multilayer bent-crystal $\mathrm{x}$-ray spectrometer. In tandem with a collimating slit, this method reveals a projection of the double differential angular-wavelength spectrum of the ICS radiation in a single shot. The measurements enabled by this diagnostic illustrate the combined off-axis and nonlinear-fieldinduced redshifting in the ICS emission process. The spectra obtained illustrate in detail the strength of the normalized laser vector potential, and provide a nondestructive measure of the temporal and spatial electron-laser beam overlap.
\end{abstract}

DOI: 10.1103/PhysRevAccelBeams.20.060701

\section{INTRODUCTION}

Inverse Compton scattering (ICS) [1-3] is an emerging technique for obtaining narrow bandwidth, highly directional $\mathrm{x}$ rays from the collision of intense relativistic electron beams and lasers. The radiation characteristics of such ICS sources are similar to those obtained from undulators in storage ringbased light sources and the spontaneous radiation from freeelectron lasers (FELs), with a qualitative difference. This is found in the scale of the periodic fields that provoke the radiation production. That is, in contrast to the centimeterperiod $\lambda_{u}$ associated with a magnetostatic undulator array that yields a resonant radiation wavelength $\propto \lambda_{u} / 2 \gamma^{2}$, in ICS one employs a counterpropagating electromagnetic wave that gives a wavelength $\sim \propto \lambda_{L} / 4 \gamma^{2}$, where $\lambda_{L}$ is the (opticalto-infrared) laser wavelength. These scale factors result from

Published by the American Physical Society under the terms of the Creative Commons Attribution 4.0 International license. Further distribution of this work must maintain attribution to the author(s) and the published article's title, journal citation, and DOI. a Doppler shift upwards in frequency by a factor $2 \gamma^{2}$, where $\gamma$ is the electron Lorentz factor, with an additional factor of 2 resulting from the laser counterpropagation in the ICS case. Thus the use of a "laser undulator" provides a path to obtaining very energetic photons, ranging from the $\mathrm{keV}$ to $\mathrm{MeV}$ using only modest energy, $20 \mathrm{MeV}$ to few-100 MeV electron linear accelerators (linacs). With smaller energies demanded, an ICS instrument is potentially very compact in size, permitting $\mathrm{X}$-ray light source research facilities in small university-scale laboratories [4]. Emerging applications in medicine $[5,6]$ that can benefit from nearly monochromatic hard $(\sim 10-100 \mathrm{keV}) \mathrm{x}$-rays are found in both diagnosise.g. in phase contrast or dual-energy digital subtraction imaging - and therapy, where $K$-edge absorption may be used to greatly enhance local x-ray dose (e.g. photon activation therapy [7]). The potential to reach subpicosecond $\mathrm{x}$-ray pulses further opens up new possibilities in ultrafast experiments, particularly in single-shot, pump-probe investigations $[8,9]$. At higher electron beam energies, in the 100's of $\mathrm{MeV}$ range [10], one may produce $\mathrm{MeV}$ photons for fundamental nuclear photonics investigations [11], or for detection of special nuclear materials [12]. 
The robustness of all the applications listed above depends, to varying degrees, on the details of the ICS spectral distribution in wavelength and angle. For a single electron radiating under the influence of a counterpropagating plane wave, the spectral dependence of scattered radiation wavelengths $\lambda_{r}$ on electron energy and emission angle can be approximately written as

$$
\lambda_{r}=\frac{\lambda_{L}}{4 \gamma^{2}}\left[1+(\gamma \theta)^{2}+\frac{1}{2} a_{L}^{2}\right] .
$$

Here $\theta$ is the emission angle measured from the electron propagation direction, and the normalized vector potential, $a_{L}=e E_{L} \lambda_{L} / 2 \pi m_{e} c^{2}$ (with $E_{L}$ the laser's electric field amplitude), measures the degree to which the electromagnetic wave induces relativistic transverse motion in the oncoming electron. This transverse motion has the effect of lowering the effective $\gamma$ of the directed longitudinal motion, and is thus sometimes referred to as the mass shift effect [13]; it is also commonly known as the nonlinear redshift. In the present work, we indicate the maximum value of $a_{L}$ that is associated with the peak value of $E_{L}$ in the laser beam as $a_{0}$.

There are finite collision-angle effects due to deviations of both the electron and laser photon trajectories [14] from the axial direction that increase the scattered bandwidth, and reduce the associated brightness. This implies that highly focused systems, while producing more photons in the collision, yield a larger spread in photon energy at a given angle. A further, fundamental constraint on source brightness arises when one focuses the laser to higher intensity, again producing more scattered photons, but concomitantly increasing the value of $a_{L}$. As seen in Eq. (1), a value of $a_{L}$ not small compared to unity causes strong spreading, proportional to $a_{L}^{2}$, of the emitting ICS spectrum, as well as novel effects due to self-interference in electron radiation processes [15-20]. An additional spectral dilution effect occurs when one approaches or exceeds $a_{L} \sim 1$, as harmonics of the radiation are produced. These phenomena serve to lower the spectral brightness of the ICS radiation produced, but may also permit development of ICS sources possessing orbital angular momentum [21]. As such, both nonlinear redshift and harmonic generation have attracted recent experimental and theoretical attention.

\section{EXPERIMENTAL DESCRIPTION}

Pursuant to the above described physics motivations, a recent experimental study performed at the Brookhaven National Laboratory Accelerator Test Facility (BNL ATF) has examined the relevant aspects of the nonlinear ICS interaction [22], with cases where $a_{L} \sim 0.6$ showing strong evidence of both redshifting, as well as harmonic scattering up to third order. These experiments $[23,24]$ employed metallic foils that exploit $K$-edge absorption to produce low-pass and bandpass filters. While these filters adequately permit isolation of harmonics and sensitive determination of the threshold for nonlinear redshifting, they do not yield detailed information on the spectral shape. Further, the correlations between angle and wavelength may only be approximately determined. As an experimental understanding of the details of the scattered spectrum is essential for applications of ICS, a more powerful diagnostic, simultaneously revealing both angular and energy spectral information is demanded. This is yet more urgent in the experimental scenario discussed below, where improvements in the laser system quality, in particular an increase of peak power from 0.4 to $0.8 \mathrm{TW}$, have yielded an unprecedented value of the normalized vector potential, with $a_{L}$ reaching near unity-or a factor of over 2.5 increase in nonlinear redshift. This context yields a rich array of observable laser-electron interaction physics that can be quantified by more insightful diagnostic techniques.

As such, we have developed and utilized in strong nonlinear-regime ICS experiments, again performed at the BNL ATF, an X-ray spectrometer [25] that is capable of revealing the double differential radiation spectrum (DDS) obtained from the scattered photons. This x-ray spectrometer, which is shown schematically in Fig. 1, consists of a collimating slit array, a bent molybdenum-silicon (Mo-Si) multilayer crystal to disperse the wavelength spectrum, and a microchannel plate (PHOTONIS MCP 40/12/10/8 I 60: 1 EDR KBR 6" FM $\mathrm{P} 46)$, in combination with a phosphor screen and a 12-bit CCD camera (Basler, SCA1400-17). The MCP and its readout form a two-dimensional position sensitive detector optimized to produce x-ray images. The bent multilayer crystal itself is constructed with a periodic Bragg structure having $45 \mathrm{Mo}-\mathrm{Si}$ pairs, each layer of thickness $20 \AA$. It reflects $\mathrm{x}$ rays in the same manner as a natural crystal with the lattice spacing of $\sim 40 \AA$, with the relation between the reflection angle and $\mathrm{x}$-ray energy given by the Bragg's law, indicated here as

$$
\sin \left(\theta_{B}\right)=\frac{0.155 m}{E_{x \text { ray }}(\mathrm{keV})},
$$

where $\theta_{B}$ is the Bragg angle and $m$ is the reflection order.

The multilayer crystal is curved into a cylindrical section [25]. The curvature permits the incidence angle of the ICS $x$ rays impinging on the crystal to vary from 25 to $10 \mathrm{mrad}$, corresponding to the $\mathrm{x}$-ray energies ranging from [Eq. (1)] 6 to $15 \mathrm{keV}$. This energy range overlaps optimally with the expected fundamental harmonic of the $\mathrm{x}$-ray spectrum in the BNL ATF ICS source. These $x$-ray energies are dispersed along the (horizontal) $x$ direction in Fig. 1, while in the orthogonal direction the mapping of reflection angle along the $y$-axis directly represents the vertical emission angle of $x$ rays from the collision point. In order to separate the angular emission from the wavelength dispersion information, previous to encountering the bent crystal 


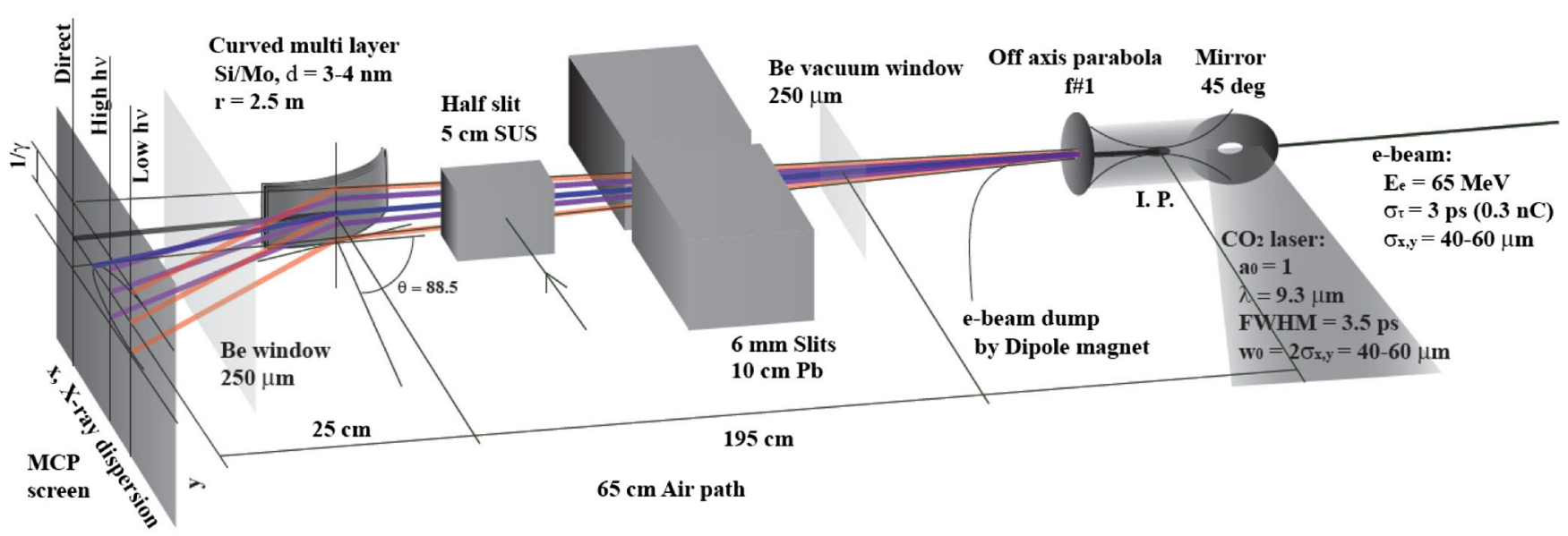

FIG. 1. Schematic layout of bent multilayer spectrometer used to obtain double differential ICS spectrum in BNL ATF experiments.

the $\mathrm{x}$ rays pass through a narrow (in the $x$ direction) slit. Thus the x-ray distribution detected and recorded at the MCP and associated apparatus closely approximates a section of double differential spectrum, $U\left(\lambda, \theta_{y}\right)$. An example of such a DDS section is shown below in Fig. 3. In the current case, where we examine the fundamental ICS harmonic generated with a linearly polarized laser, the angular spectrum is not exactly axially symmetric for large $a_{0}$, being elongated along the direction of the polarization. Thus the section of the DDS $\left.U\left(\lambda . \theta_{y}\right)\right|_{\theta_{x}=0}$ is marginally dependent on the relative direction of the laser polarization and the slit direction;

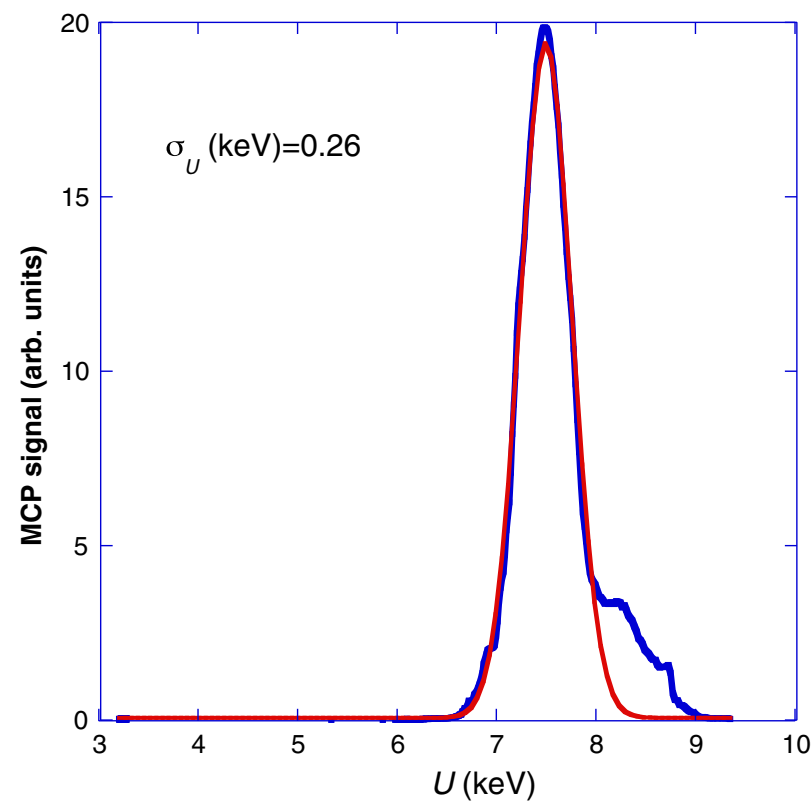

FIG. 2. Results of calibration tests of spectrometer at BNL NSLS-I X15A (in blue), measured dispersion (photon energy vs radiation angle $x$ ), displaying a resolution at $7.5 \mathrm{keV}$ central energy of $0.26 \mathrm{keV}$ (rms), as illustrated by Gaussian fit to peak (in red). in the experiments reported here the slit and polarization directions are at 30 degrees with respect to each other. For the following discussion, which concentrates mainly on the near-axis component to analyze nonlinear redshift effects, this polarization direction does not affect the spectrum.

The calibration of the mapping of dispersive angle to $\mathrm{x}$-ray energy in this instrument was accomplished using known photon energy distributions at a number of different central energies obtained from the NSLS x-ray light source beam line X15A, using a Si 111 and 333 monochromator having resolution $\Delta \nu / \nu=2 \times 10^{-4}$, and a hard x-ray CCD BAS2500 image plate reader. The rms resolution of the bent crystal spectrometer deduced from this calibration procedure is $0.26 \mathrm{keV}$ at a central energy of $7.5 \mathrm{keV}$, in the middle of the spectral band of interest. We note that the fractional resolved bandwidth is approximately the inverse of the number of Bragg layers.

To create the scattered photons, a similar set of experimental conditions was used at the BNL ATF ICS source as was employed in the experiments of Ref. [22], with the notable exception of an increase in laser peak power. In the experiments reported here the electron beam parameters were: $\sim 3$ ps rms pulse length, $\sim 1.3 \mathrm{~mm}$-mrad normalized emittance, $0.3 \mathrm{nC}$ total charge, and $E_{0}=65 \mathrm{MeV}$. This beam is generated by an rf photoinjector, accelerated in a following linac, and subsequently transported and focused to $\sim \sigma_{e}=40 \mu \mathrm{m} \mathrm{rms}$ spot where it is collided with a $\sim 3.5$ ps FWHM $\mathrm{CO}_{2}$ laser [26] $\left(\lambda_{L}=9.3 \mu \mathrm{m}\right)$. We note that the electron beam focus is sufficiently relaxed that, given the emittance, the induced bandwidth from electron angles during collision is small, as discussed further below. This laser pulse has between 1.5 and $3.0 \mathrm{~J}$ of total energy, corresponding to $\sim 0.4-0.8 \mathrm{TW}$ peak power, and with $w_{0}=2 \sigma_{L} \approx 60 \mu \mathrm{m}$, normalized vector potentials up to $a_{0}=1$ are obtained. The electrons are brought into collision with the laser by use of strong quadrupole focusing magnets and an alignment pinhole. The electron beam rms size at focus is significant in determining the 


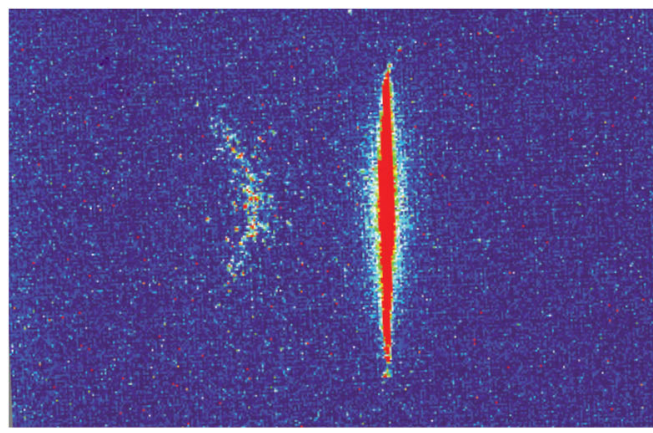

(a)

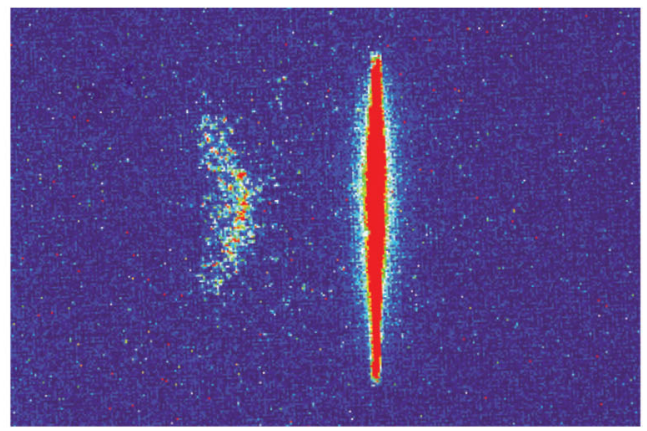

(b)

FIG. 3. (a) ICS photon distribution for $1.5 \mathrm{~J}$ laser interaction $\left(a_{L}=0.7\right)$, after passage through bent crystal, showing both undiffracted (vertically oriented bright red spot) and diffracted (dispersed) component; (b) higher laser energy case, with $3.0 \mathrm{~J}$ and $a_{0}=1$. Note the curved shape of the diffracted double differential spectrum in both images, as well as the enhanced width due to nonlinear redshifting in the higher laser energy case.

spectral shape of the ICS radiation, as the condition $\sigma_{e} \approx \sigma_{L}$ produces a notable experimental spectral signature, as discussed below. Temporal synchronization of the electrons and laser is measured and optimized via use of a $\mathrm{Ge}$ semiconductor switch [27] located at the laser- and electron-beam interaction point. The ICS x-rays are extracted through a $250 \mu$ m-thick Be window and $0.5 \mathrm{~m}$ air path before passing through the bent multilayer spectrometer.

\section{RESULTS AND DISCUSSION}

Two images of the ICS x-rays obtained after the spectrometer and slit apparatus are shown in Fig. 3, corresponding to the partially and strongly nonlinear cases with $1.5 \mathrm{~J}\left(a_{0}=0.7\right)$ and $3.0 \mathrm{~J}\left(a_{0}=1\right)$ laser, respectively. In these images the main features of the projected ICS DDS $U\left(\lambda, \theta_{y}\right)$ are displayed. First, there is a curved intensity distribution corresponding to the parabolic off-axis redshifting indicated by the $\theta$-dependence shown in Eq. (1). To illustrate this dependence, we show in Fig. 4 the spectral edges $(15 \%$ integrated intensity from the minimum and maximum energy limits of the observed x-ray distributions, respectively) as a function of emission angle $\theta_{y}$, corresponding to the $a_{0}=1$ laser case in Fig. 3. As can be seen from the data and the predictions of the particle trackingbased, Liénard-Wiechert (L-W) simulations [22] of the experimental scenario, utilizing Gaussian laser intensity and electron beam density profiles (whose physical significance is examined below), one observes a very large bandwidth spectrum at a given $\theta_{y}$, even for the less energetic, lower $a_{0}$ case. The details of the wavelength spectral shape at constant $\theta_{y}$ revealed in this DDS image are central to the current investigation, and are analyzed further in the following discussion.

In order to appreciate the physics processes involved in the creation of the wavelength spectral distribution shape due to the intense-laser induced nonlinear red (mass shift) shifting effect, some theoretical discussion is warranted. We begin by examining the broadening of the spectral
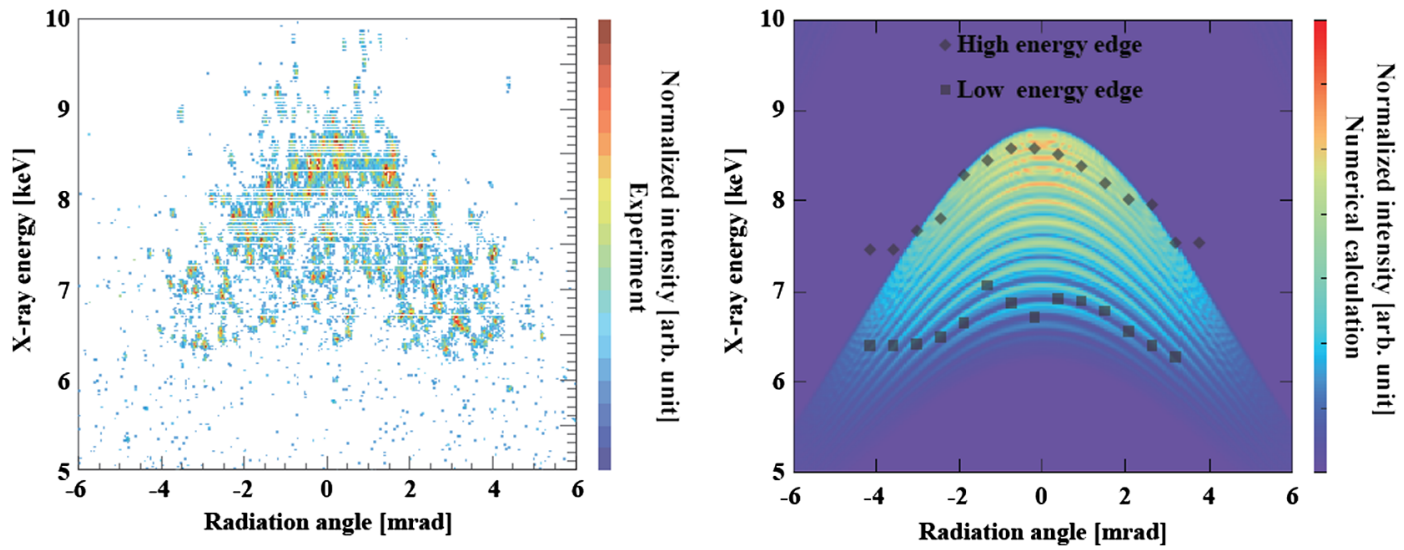

FIG. 4. (left) Calibrated ICS DDS data through $2 \times 250 \mu \mathrm{m}$ Be windows and $65 \mathrm{~cm}$ air path, $3 \mathrm{~J}$ laser energy case, using bent crystal system, from Fig. 3. (right) Analysis of angularly resolved energy distribution of ICS x-rays from measurement. For a given $\theta_{y}$, high energy and low energy edges (15\% integrated intensity) are shown. Predictions of the Liénard-Wiechert calculated spectral intensity are displayed with a color map. 
distribution at a given $\theta_{y}$ due to the nonlinear redshifting process. In analyzing these spectra, we initially deemphasize the fine structure, displayed in Fig. 4(b), that is introduced in the spectral shape by interference effects $[15,18,28]$ arising from the time dependence of the emission process [29]. Such effects are challenging to resolve in the experiments with the present spectrometer, a point we return to later. We thus concentrate for the moment on the local changes in amplitude of the electric field in the laser and their effects on the nonlinear redshift.

Theoretically, this model is equivalent to assuming that both the local Thomson scattering probability, and nonlinear redshifting of the scattered photon produced with this probability, are proportional to the laser intensity, $a_{L}^{2} \propto I$. This approximation ignores the wave-phase derived effects observed in the Liénard-Wiechert model and in more sophisticated theoretical treatments [29]. We assume for simplicity that the distribution of laser intensity is Gaussian both in the temporal and radial dimensions; likewise we also assume that the electron beam has a Gaussian transverse distribution of rms width $\sigma_{e}$. In this analysis, we also assume that the electron transverse position does not notably change during the laser-electron collision. This condition is well obeyed, as the minimum beam $\beta$-function, which measures the transverse spreading scale length of the electron beam, is $\sim 4 \mathrm{~cm}$; this is much longer than the interaction length. We further assume that we can ignore diffraction effects in the laser focus during collision, thus requiring (for Gaussian temporal pulse profiles) $Z_{R}>c T_{L} / 4.7$. Here we have a laser Rayleigh range $Z_{R}=0.5 \mathrm{~mm}$, and spatial pulse length (FWHM) $c T_{L}=0.22 \mathrm{~mm}$, and the approximation holds well.

The structure of the ICS spectral distribution indeed arises in part from the time variation in the intensity, parametrized by $a_{L}^{2}(t)$, which as implied above is viewed in good approximation as a property of the laser alone in the case of head-on laser-electron beam collisions. The ICS spectral shape is also due to the transverse variation of the laser field. The degree to which this effect asserts itself is dependent on the parameter $\kappa \equiv\left(\sigma_{L} / \sigma_{e}\right)^{2}$, the ratio of the electron beam rms transverse spot area to that of the laser beam. For $\kappa \gg 1$, the transverse variation in the field is not significant, but we shall see that for $\kappa \sim 1$ or below, transverse field variations are notable and the ICS spectral shape is strongly affected. This is indeed the case for the present experiments, and thus the measurement of the spectral shape at constant $\theta_{y}$ permits experimental probing of the overlap between the electron and laser beam distribution, i.e. an independent, nondestructive determination of $\kappa$.

In the case of a temporal and transverse spatial, axially symmetric bi-Gaussian laser pulse, with the assumed intensity distribution

$$
I(r, t) \cong I_{0} \exp \geq\left[-\frac{r^{2}}{2 \sigma_{L}^{2}}\right] \exp \left[-\frac{t^{2}}{2 \sigma_{t}^{2}}\right],
$$

we can obtain analytical estimates for the spectral shape that yield information on the spatial-temporal characteristics of the interaction. We review the main results of this analysis below; the derivation of these results is given in detail in the Appendix.

Concentrating first on the temporal dependenceassuming no transverse variation in the intensity, nor any other contributions to homogeneous spectral broadening (i.e., energy spread, electron or laser photon angles) - the on-axis $\left(\theta_{y}=\theta_{x}=0\right)$ spectral shape is described, following Eq. (1), by the distribution

$$
P_{1}(\Delta \lambda)=\frac{C_{1}}{\sqrt{\operatorname{In}\left(\Delta \lambda_{\max } / \Delta \lambda\right)}},
$$

where $\Delta \lambda$ is the nonlinear redshifting, $C_{1}$ is a normalization constant, and the distribution function is weakly divergent near the maximum nonlinear redshift, $\Delta \lambda_{\max }=\lambda_{L} a_{0}^{2} / 8 \gamma^{2}$. This divergence is of course eliminated in practice by homogeneous broadening effects. The predictions of this straightforward, heuristic scattering model are consistent with the results of a full L-W calculation [22], as displayed in Fig. 5, which uses the maximum photon energy and redshift parameters encountered in the scenario of Figs. 3(b) and 4.

The overall spectral shape is reproduced well, but by construction lacks the oscillations, often referred to in the literature as subsidiary peaks, due to the self-interference effects described above. As these artifacts have drawn considerable theoretical interest in the recent past, and we will discuss below their role in the experiments, it is worth clarifying the various theoretical viewpoints concerning this spectral structure, which uniquely arises in nonlinear ICS. Descriptions of this spectral structure were initially reported in 2004 by Brau in Ref. [16] and by Krafft in Ref. [15]. The number of subsidiary peaks expected in the spectrum was first empirically estimated for a single laser wavelength by Heinzl et al. in Ref. [18]. An expression for the number of peaks in this spectrum for an arbitrary wavelength and normalized vector potential was derived by Terzic et al. in Ref. [19], and the manipulation of nonlinear ICS spectra by use of laser frequency chirping was proposed. In 2016, Rykovanov et al. [28] proposed an approximate method to determine the number of spectral peaks and discussed further the issue of frequency chirping. This work was performed roughly contemporaneously with the detailed treatment by Terzić, Reeves, and Krafft [20], which extended the analysis to cover harmonics. A discussion followed in Ref. [30] that provided a resolution of the issues pertaining to the number of subsidiary peaks, and to employing laser frequency chirps for minimizing ICS bandwidth.

The theoretical work quoted above concentrates on the case where the only variation in the laser strength examined is in the longitudinal direction. In experiments, however, 

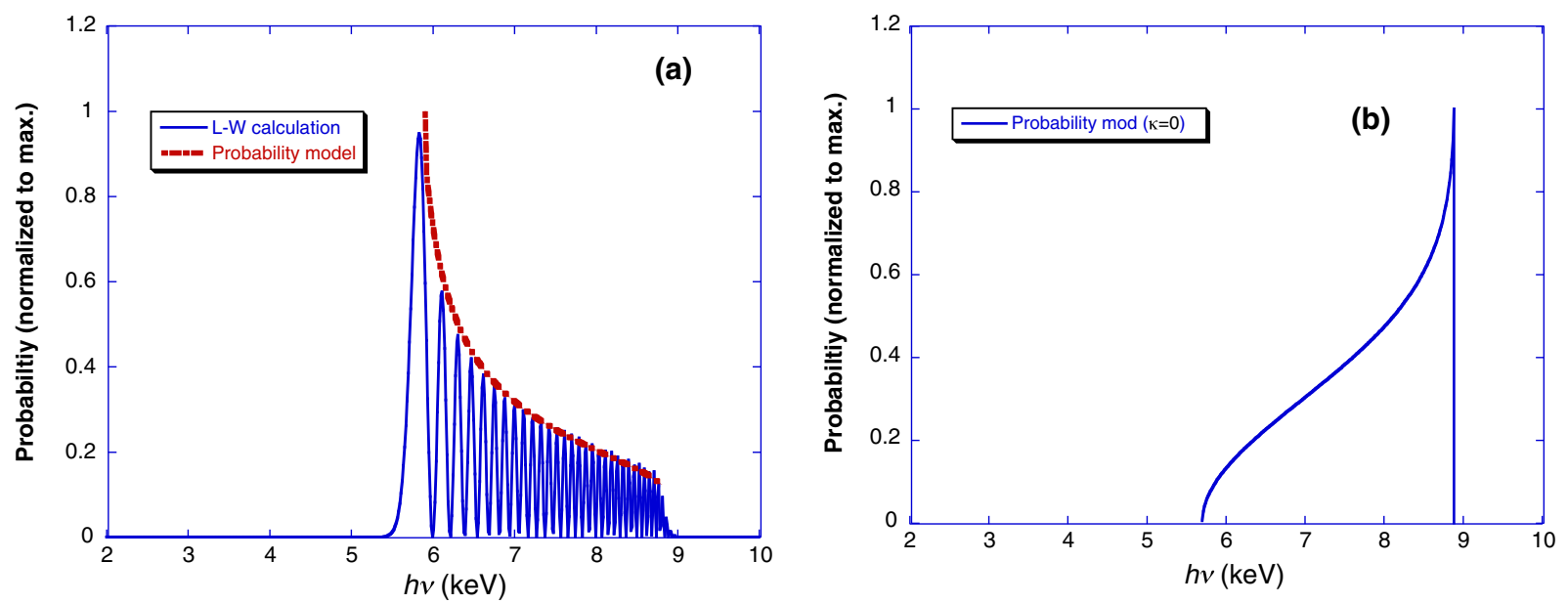

FIG. 5. (a) ICS spectrum considering only a longitudinal field variation (maximum photon energy $h \nu_{\max }=8.7 \mathrm{keV}, a_{0}=1$, maximum redshift $2.9 \mathrm{keV}$ ) from probability model (red dashed). Also shown, the ICS spectrum as calculated by the L-W model, which displays self-interference derived oscillations in this spectral intensity. (b) The prediction of the probability model when the transverse field variations are dominant $\left(\kappa=0\right.$ limit, with $\left.h \nu_{\max }=8.7 \mathrm{keV}, a_{0}=1\right)$, showing the peak in spectrum at minimum redshift.

one must focus the laser strongly to maximize the scattered photon production; in the process of focusing, one also maximizes the redshifting effects that we wish to study here. Thus there is a notable variation in laser intensity in the transverse direction due to optimization to smaller laser spot sizes. This effect, we shall see, lowers the peak intensity in the ICS spectrum near maximum nonlinear redshifting indicated in Eq. (4) and in Fig. 5(a). To give insight into this, we analyze the probability associated with the change in laser intensity off axis at a radial position $r$ combined with the probability of finding an electron at the same radius. Here we ignore for the moment the longitudinal (temporal) variation of the field discussed above. In this analysis, we take into account the effect of the weighting of the probability with radius $r$ as well as the possibility of differing beam sizes, i.e. different values of $\kappa$, which here serves as a parameter classifying the spectral shape. This analysis (see the Appendix) yields a rather simple relation for the probability of finding the redshift condition $\Delta \lambda$,

$$
P_{2}(\Delta \lambda)=C_{2}\left(\frac{\Delta \lambda}{\Delta \lambda_{\max }}\right)^{\kappa-1}
$$

with $C_{2}$ also indicating a normalization constant. Note that for $\kappa>1$, the probability distribution is again found to favor large nonlinear redshift $\Delta \lambda$, but for small laser beam sizes $\kappa<1$, smaller redshifts are more probable. For $\kappa=0$, the probability distribution is dominated by the effect of transverse variation in laser intensity; for $\kappa \gg 1$, Eq. (5) approaches a $\delta$-function at $\Delta \lambda=\Delta \lambda_{\max }$.

In analyzing experiments, we wish to include both longitudinal and transverse effects to find a probability of observing a certain redshift $\Delta \lambda$. To do so we must convolve Eqs. (4) and (5), treating the total probability associated with Eq. (5) as yielding the maximum local redshift $\Delta \tilde{\lambda}$ given by all elements of the statistical ensemble indicated by differing $r$ values. In this regard, it should be noted that $P_{2}$ has not been weighted by the scattering probability $a^{2}$, as this effect is included in the convolution with the probability $P_{1}$, which contains this weighting.

This convolution is written as

$$
\begin{aligned}
P_{t}(\Delta \lambda) & =C_{t} \int_{\Delta \lambda}^{\Delta \lambda_{\max }}\left(\frac{\Delta \tilde{\lambda}}{\Delta \lambda_{\max }}\right)^{\kappa-1} \frac{1}{\sqrt{\operatorname{In}[\Delta \tilde{\lambda} / \Delta \lambda]}} d(\Delta \tilde{\lambda}) \\
& =\frac{2 C_{t}}{\Delta \lambda_{\max }}\left(\frac{\Delta \lambda}{\Delta \lambda_{\max }}\right)^{\kappa} \int_{0}^{\sqrt{\operatorname{In}\left(\Delta \lambda_{\max } / \Delta \lambda\right)}} \exp \left(\kappa u^{2}\right) d u \\
& =\frac{2 C_{t}}{\Delta \lambda_{\max }}\left(\frac{\Delta \lambda}{\Delta \lambda_{\max }}\right)^{\kappa} \sqrt{\frac{\pi}{4 \kappa}} \operatorname{erfi}\left[\sqrt{\left.\kappa \operatorname{In}\left(\frac{\Delta \lambda_{\max }}{\Delta \lambda}\right)\right]},\right.
\end{aligned}
$$

with $C_{t}$ again indicating a normalization constant. To illustrate the limit of a very narrow laser beam, where the field variation in the transverse dimension is maximized, we examine the case $\kappa \rightarrow 0$, and obtain the asymptotic expression

$$
P_{t}(\Delta \lambda)_{\kappa=0}=\frac{2 C_{t}}{\Delta \lambda_{\max }} \sqrt{\operatorname{In}\left(\frac{\Delta \lambda_{\max }}{\Delta \lambda}\right)},
$$

which gives an opposite spectral dependence compared to that of Eq. (4), peaked at small $\Delta \lambda$. This behavior is shown in Fig. 5(b). For values of $\kappa$ near unity, as is the case for the present experiments, a transition between the two limiting behaviors occurs. The spectral peak is broader for such cases, and found at an intermediate point between very low and maximum $\Delta \lambda$, as can be seen from exploration of 


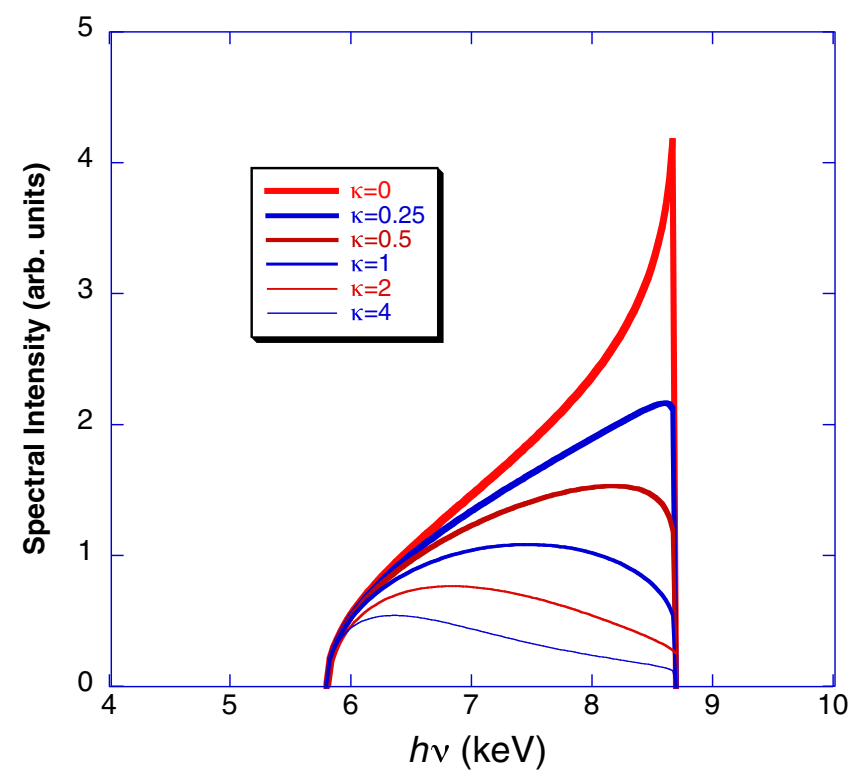

FIG. 6. ICS spectrum with maximum photon energy $h \nu_{\max }=8.7 \mathrm{keV}, a_{0}=1$ (maximum redshift $2.9 \mathrm{keV}$ ) from probability model convolution of Eq. (6), for varying ratios of laser to electron beam size: $\kappa=0,0.25,0.5,1,2$, and 4 .

Eq. (6). As can be ascertained from Fig. 6, the convolution leaves the highest redshift probabilities unchanged as $\kappa$ is varied, while the probability of having a lower redshift goes down as the laser size increases relative to that of the electron beam, eventually reaching the result obtained in Eq. (4). The comparison between probability and the L-W models has been found to be in excellent agreement for values of $\kappa$ ranging from 0.1 to 4 .

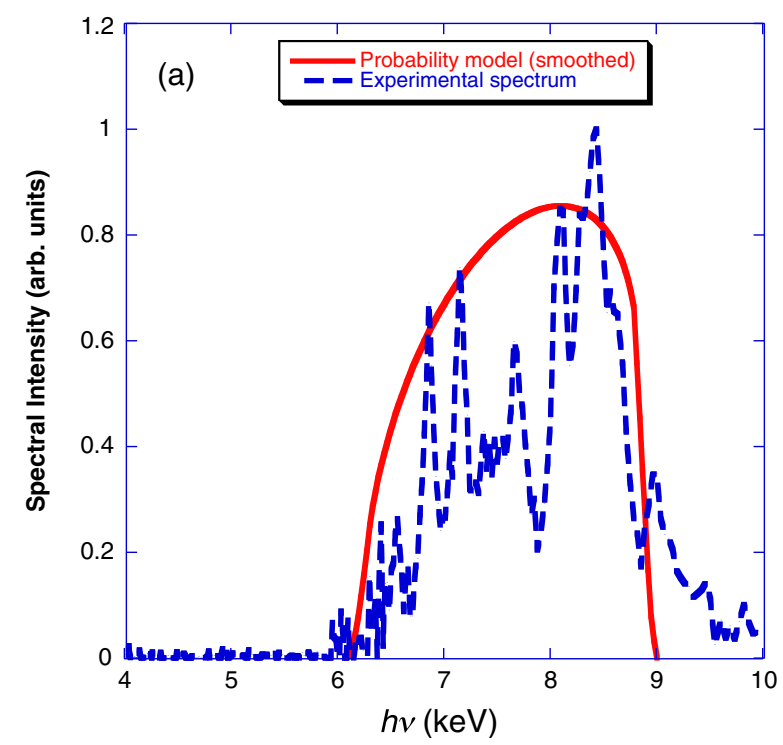

We are now in a position to examine the near-axis spectrum obtained from the maximum-intensity laser case obtained from the data given in Fig. 3(b). These data are obtained from the images in Fig. 3(b) using the established calibration between angle and photon energy $h \nu$. A nearly on-axis section of the DDS having a width in $\theta_{y}$ between $\pm 0.5 \mathrm{mrad}$ is extracted, thus permitting the off-axis redshift (at maximum $35 \mathrm{eV}$ ) to be neglected. The wavelength dependent absorption of $\mathrm{x}$-rays during transport is corrected for in the resulting spectrum.

This spectrum is shown in Fig. 7(a), along with the probability model spectrum using $\kappa^{0.5}=0.75$, and smoothed by including resolution-limiting effects, i.e. the estimated homogeneous broadening effects in the ICS spectrum as well as the calibrated resolution in the measurement system deduced from Fig. 2. The measurement system-derived rms broadening dominates at $260 \mathrm{eV}$; the broadening contributions due to the angles in the electron beam, and to the finite laser pulse length are both $\sim 10 \mathrm{eV}$, respectively. Also given in Fig. 7(b) are the predictions of the L-W model, obtained using measured laser and electron beam parameters, with $\sigma_{e}=40 \mu \mathrm{m}$, and $\sigma_{l}=30 \mu \mathrm{m}$, as noted above. The L-W calculation, which does not suffer from weakly divergent spectral artifacts, directly omits the measurement-specific spectral broadening effects. As such, the measurement resolution effects must be convolved with the L-W numerical results, with the resultant line shape also shown in Fig. 7(b).

As expected, the observed spectrum extends from above $8.7 \mathrm{keV}$ to less than $6 \mathrm{keV}$, due to the large maximum relative redshift $\Delta \lambda_{\max } / \lambda_{r 0}=\frac{1}{2} a_{0}^{2}=0.5$, or 0.33 in terms of relative photon energy referred to the maximum. Further,

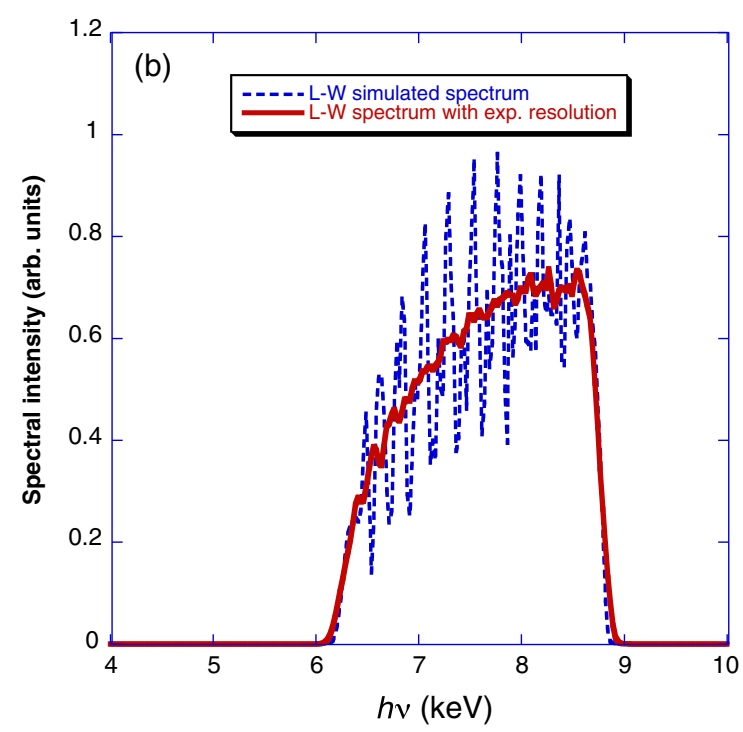

FIG. 7. (a) Observed near-axis radiation spectrum extracted from DDS image in Fig. 3(b) (blue dashed line). Also shown, predictions of probability model assuming $U_{b}=65 \mathrm{MeV}, \kappa^{0.5}=0.75, a_{0}=1$ (red solid line, with predications smoothed to reflect determined Gaussian resolution, per Fig. 2). (b) Simulated spectrum from the L-W calculation with the same parameters, showing self-interference effects (blue dashed line). Also shown, the L-W spectrum smoothed for experimental resolution (red line). 


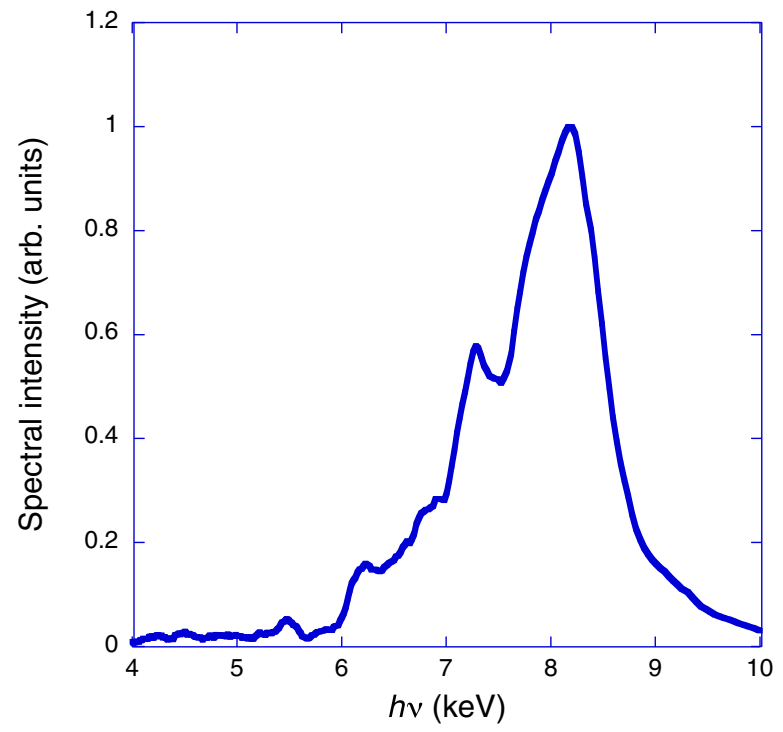

FIG. 8. Spectral shape of near-axis multiple-shot radiation, for experimental conditions near to those of Fig. 7(a).

the probability model and experimental data have similarities in spectral shape, descending from a larger spectral intensity nearer to the smallest redshift to slightly lower intensity near the largest redshift, and agreeing well in the displayed upper and lower edges - as previously noted in the spectral edge determinations in Fig. 4.

The single-shot, near-axis spectrum shown in Fig. 7(a) displays significant structure at the level of $0.15-0.3 \mathrm{keV}$, a subject we return to examine below. In order to study the overall profile of the line more quantitatively, however, we show in Fig. 8 the sum of many shots having similar laser energy, near $3 \mathrm{~J}$. The result is significantly smoothed effectively eliminating the subsidiary peak structure. We use this smoothed spectrum to find a maximum intensity, found to be near to $8.1 \mathrm{keV}$, which is in turn used to give a spectral determination of $\kappa^{0.5}$. We compare this value of $\kappa^{0.5}$ with that obtained by electron and laser beam spot size measurements. In Fig. 9, we show the predicted peak locations from the probability model as a function of $\kappa^{0.5}$ for the known beam energy and nominal laser intensity. It can be seen that the experimental maximum determines a spectrally derived value of $\kappa^{0.5}$ near 0.82 , with lower and upper limits derived from the photon energy resolution of 0.70 and 0.92 , respectively. These results are consistent with the direct measurement of electron and laser beam sizes, $\kappa^{0.5}=0.75 \pm 0.10$.

The data from the spectrometer measurement display some deviations from the probability model description. The observed spectral peak is sharper and at slightly higher energy than predicated by the probability model, discrepancies that are partly due the laser and electron beam profiles deviating from Gaussian distributions. In particular, both transverse electron beam as well as halo may emphasize smaller redshift photon production. Further, the

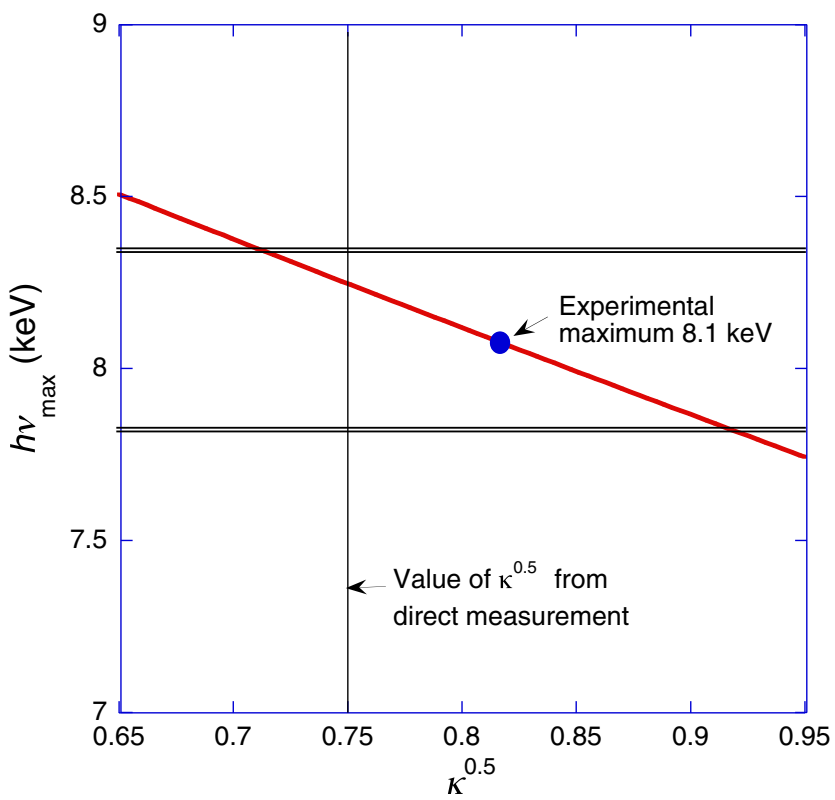

FIG. 9. Theoretically predicted locations of the spectral maximum as a function of $\kappa$ (solid red line). Also shown, the experimentally observed location of the maximum at $8.1 \mathrm{keV}$, placed to intercept the theoretical prediction (red line) at $\kappa^{0.5} \sim 0.82$. Also shown are the upper and lower limits (double lines) on the energy of maximum spectral intensity obtained from the system spectral resolution, $0.70<\kappa^{0.5}<0.92$, and the value from electron and laser beam measurements, $\kappa^{0.5}=0.75$.

laser beam also possesses both satellite pulses and pedestal, both of which function to produce more photons at smaller redshift. Finally, the laser beam measurements at the interaction point are performed without full amplification, and some differences in properties are expected at high power. These issues point to the utility of an independent, nondestructive determination of $\kappa$. Refinement of this method, using a more detailed model of expected laser and electron beam profiles is clearly both possible and desirable.

The spectral data shown in Fig. 7(a) and the simulated L-W spectrum in Fig. 7(b) also display quasiperiodic peaks. In the case of simulation, it is known that they are associated with self-interference effects. It is interesting to ask whether these are related to those found in the experimental spectra. Indeed, Fourier analyses of both the experimental spectra and L-W predictions yield similar characteristics, displaying oscillations with approximately $0.3 \mathrm{keV}$ periods each (or $\sim 12$ peaks inside of the spectral distributions). In the theoretical treatment, the scale of this period is set by the interference condition, which is a function maximum nonlinear redshift and the laser pulse length [19]. Care must be taken in evaluating the number of periods from the theoretical predictions, which concern only temporal variation effects. Indeed, the concise result of Ref. [19] indicates that over 60 subsidiary peaks should be present, which is reproduced in simulations having only 
temporal variations, but not in the simulation where radial variations in the beams are present [Fig. 7(b)]. In order to extend the theoretical prediction for these peaks, it would be necessary to convolve the probabilities associated with the radial variations of interaction parameters, in analogy to what is done above and in the Appendix. These effects are of course included automatically in the L-W simulation; the inclusion of radial variations changes the number of peaks, and also diminishes their contrast - the minima between the peaks does not reach zero in Fig. 7(b).

The measured and computationally predicted periods of the observed peaks are both only a bit larger than the experimentally determined resolution of the curved multilayer, $\sigma_{E}=0.26 \mathrm{keV}$, however. To show the likelihood that the interference effect would be definitively detected, the spectrometer resolution effect is convolved with the L-W simulation predictions, as shown in Fig. 7(b). The oscillations in the spectrum are seen to barely survive in this simulation of the measurement. Thus this aspect of the nonlinear ICS physics was not likely to be (and certainly not definitively) observed in the experiment as it was performed. Any apparent oscillations are probably due to the granular nature of the obtained spectrum arising from the finite number of photons detected at the MCP and associated screen, a phenomenon displayed in Fig. 4. The statistical sampling of the data has been improved by taking the maximum width of the DDS in angle consistent with being able to ignore the off-axis redshift, but there are still notable statistical fluctuations in the data, as displayed in Fig. 7(a).

\section{CONCLUSIONS}

The results of measurements obtained from use of the curved multilayer spectrometer described here thus reveal, on a single-shot basis, compelling details of the double differential ICS spectrum in the highly nonlinear regime where $a_{0}$ reaches unity. The results of these measurements clearly illustrate the redshifting effects associated with offaxis emission and nonlinear electron motion in the large laser field in the fundamental emitted band. Further, the shape and width of the ICS near-axis photon energy spectrum are seen to give quantitative insight, within experimental error, into the relative laser-electron beam spots $\kappa$, as well as the degree of nonlinearity parametrized by $a_{0}$. The methodology employed here, as well as the push to considerably higher laser intensities $(\sim 2.5$ times larger than those of Ref. [22]), thus provides a much more detailed look into the electrodynamic processes involved in nonlinear inverse Compton scattering than previously obtained. As such, this investigation has, importantly, introduced a powerful new experimental method for understanding the intense laser-relativistic electron beam interaction, and also in the process also shed considerable light on the spectral structure of ICS in this limit, which is highly relevant to applications. Indeed, the vigorous theoretical discussion of the ICS spectrum in the nonlinear regime for over a decade now points out the utility of this single-shot spectrometer-this experimental tool would be essential for investigating the efficacy of chirped laser effects in the ICS interaction. This type of spectrometer may be used for photons up to $100 \mathrm{keV}$ by using a natural crystal such as $\mathrm{LiF}$ instead of a multilayer [31]; such an experimental effort is currently under development.

Returning to the issue of self-interference in ICS, an effect that has been inconclusively resolved by use of the current bent crystal dispersive system, the method introduced here may be further improved to allow detection of this intriguing phenomenon. To investigate these effects, one may optimize the design of the spectrometer to improve the spectral resolution $[32,33]$. This improvement can be accomplished by using thinner, more numerous multilayer strata with attendant change in incident angle. It will also be of interest, in the highly nonlinear $a_{L} \sim 1$ limit, to examine the double differential spectrum associated with ICS higher harmonics in the strong laser field. Experiments are also planned that examine two-laser-frequency ICS, in which a scattered spectrum that contains significant subsidiary peak structure is produced; sidebands of the low $a_{L}$, high $\omega_{L}$ laser are introduced by the presence of a high $a_{L}$, low $\omega_{L}$ laser. Such issues may be taken up in future experiments that will seek to fully exploit the methods introduced in the present work.

\section{ACKNOWLEDGMENTS}

This work supported by the U.S. DOE Contract No. DESC0009914, the U.S. Department of Homeland Security Grant No. 2014-DN-077-ARI084-01, and by the National Science Foundation under Grant No. PHY-1549132.

\section{APPENDIX: THE SCATTERING PROBABILITY MODEL}

The designation of the process of light scattering from relativistic electrons, while commonly known as inverse Compton scattering, is at the energies of interest better described as Thomson scattering, as in the nominal electron rest frame the Doppler shifted energy of the laser photon is small, $\hbar \omega \ll m_{e} c^{2}$. Thus one may neglect the electron recoil, leading to a scattered spectrum given by Eq. (1), which is the Compton spectrum obtained in the Thomson limit. If one adopts the viewpoint of particlelike scattering inherent in the Compton/Thomson description, then in this limit the Thomson cross section $\sigma_{T}$, when multiplied by the density of the laser photons, gives an excellent approximation of the probability of a scattering event. This scattering is implicitly viewed as dependent only on instantaneous photon density, and by definition does not contain information on wave effects such as the interference discussed above.

One may thus, based on this local scattering probability, obtain the associated scattered spectral probability 
distributions specific to the temporal and spatial distributions of laser and electron beams. Under the relevant experimental conditions, these effects may be analyzed separately. We first examine the effect of the temporal distribution of laser photons, reserving the more subtle analysis of the spatial distributions for discussion below.

In the case of the temporal distribution alone, we examine the laser probability, assuming all electrons experience the same temporal shape of the laser pulse. For this analysis to be valid we require explicitly that the interaction time of the laser pulse (with an assumed Gaussian intensity shape of width $\sigma_{t}$ ) is shorter than the time to traverse the laser focus Rayleigh length, $c \sigma_{t} \ll Z_{R}$. We begin by normalizing the probability of measuring a time as a priori equal over some arbitrary temporal period $T \gg \sigma_{t}$, and the probability is simply $T(t)=1 / T$. As the square of the vector potential follows the laser is identical to the intensity, $a^{2}(t)=a_{0}^{2} \exp \left\lfloor-t^{2} / 2 \sigma_{t}^{2}\right\rfloor$, the probability of encountering a given value of $a^{2}$ in the laser pulse is derived from $A\left(a^{2}\right) d a^{2}=T(t) d t$, or

$$
A\left(a^{2}\right)=\frac{\mathrm{T}(t)}{\frac{d a^{2}}{d t}}=\frac{\sigma_{t}^{2}}{T t \exp \left[-t^{2} / 2 \sigma_{t}^{2}\right]} .
$$

With $t / \sigma_{t}=2 \sqrt{\operatorname{In}\left(a_{0} / a\right)}$, this probability is compactly written as

$$
A_{t}\left(a^{2}\right)=\frac{\sigma_{t}}{T} \frac{a_{0}^{2}}{a^{2}} \frac{1}{2 \sqrt{\operatorname{In}\left(a_{0} / a\right)}} .
$$

Now, the probability of scattering a photon with this value of $a^{2}$ is thus simply

$$
N_{t}\left(a^{2}\right)=a^{2} A_{t}\left(a^{2}\right)=\frac{\sigma_{t}}{T} \frac{a_{0}^{2}}{2 \sqrt{\operatorname{In}\left(a_{0} / a\right)}} .
$$

In terms of redshifted wavelength, then, one may simply write

$$
P_{1}(\Delta \lambda)=\frac{C_{1}}{\sqrt{\operatorname{In}\left(\Delta \lambda_{\max } / \Delta \lambda\right)}} .
$$

To construct a full probability model including all effects of the shape of both the electron and the laser distribution, we must now look at the spectral probability associated with these assumed radially symmetric distributions. is

The probability of finding an electron at a given radius $r$

$$
R_{e}(r)=\frac{r}{\sigma_{e}^{2}} \exp \left(-r / 2 \sigma_{e}^{2}\right)
$$

while the dependence of the vector potential on $r$ is where $a^{2}(r)=a_{0}^{2} \exp \left\lfloor-r^{2} / 2 \sigma_{l}^{2}\right\rfloor$, where $\sigma_{e}$ and $\sigma_{l}$ are the radial Gaussian widths of the electron and laser beams, respectively. The probability of an electron experiencing the normalized vector potential $a^{2}$ is thus

$$
\begin{aligned}
A_{r}\left(a^{2}\right)=\frac{R_{e}(r)}{\left|\frac{\partial a^{2}}{\partial r}\right|} & =\frac{\sigma_{l}^{2}}{\sigma_{e}^{2} a^{2}} \exp \left(-\frac{r^{2}}{2 \sigma_{e}^{2}}\right) \\
& =\frac{\sigma_{l}^{2}}{\sigma_{e}^{2}}\left(\frac{a^{2}}{a_{0}^{2}}\right)^{\kappa}=\frac{\kappa}{a_{0}^{2}}\left(\frac{a^{2}}{a_{0}^{2}}\right)^{\kappa-1},
\end{aligned}
$$

where we have defined $\kappa \equiv \sigma_{l}^{2} / \sigma_{e}^{2}$, and used $\exp \left(-\frac{r^{2}}{2 \sigma_{e}^{2}}\right)=$ $\left(\frac{a^{\frac{\sigma_{l}^{2}}{2}}}{a_{0}^{2}}\right)^{\frac{\sigma_{e}^{2}}{\sigma_{e}}}=\left(\frac{a^{2}}{a_{0}^{2}}\right)^{\kappa}$. Equation (A6) may be rewritten explicitly in terms of the redshifted wavelength as

$$
P_{2}(\Delta \lambda)=C_{2}\left(\frac{\Delta \lambda}{\Delta \lambda_{\max }}\right)^{\kappa-1} \text {. }
$$

Note that we have not weighted this redshift probability by the scattering probability $a^{2}$, as we intend to use it convolved with the probability $P_{1}$, which already has this weighting.

Formally, the convolution of the temporal scattered photon spectral distribution Eq. (A4) with the probability is thus

$$
P_{t}(\Delta \lambda)=C_{t} \int_{\Delta \lambda}^{\Delta \lambda_{\max }} P_{2}(\Delta \tilde{\lambda}) P_{1}(\Delta \tilde{\lambda}) d(\Delta \tilde{\lambda})
$$

In Eq. (A8) we are summing over the spectral probabilities $P_{1}(\Delta \tilde{\lambda})$ with the local (for a given value of $r$ ) weight $P_{2}(\Delta \tilde{\lambda})$ corresponding to the local maximum redshift at the location $r$. This produces the expression

$P_{t}(\Delta \lambda)=C_{t} \int_{\Delta \lambda}^{\Delta \lambda_{\max }}\left(\frac{\Delta \tilde{\lambda}}{\Delta \lambda_{\max }}\right)^{\kappa-1} \frac{1}{\sqrt{\operatorname{In}(\Delta \tilde{\lambda} / \Delta \lambda)}} d(\Delta \tilde{\lambda})$.

Equation (A9) is analyzed further in Eq. (6) in the text.

[1] G. R. Blumenthal and R. J. Gould, Synchrotron radiation, and compton scattering of high-energy electrons traversing dilute gases, Rev. Mod. Phys. 42, 237 (1970).

[2] P. Sprangle, A. Ting, E. Esarey, and A. Fisher, Tunable, short pulse hard x-rays from a compact laser synchrotron source, J. Appl. Phys. 72, 5032 (1992).

[3] W. P. Leemans, R. W. Schoenlein, P. Volfbeyn, A. H. Chin, T. E. Glover, P. Balling, M. Zolotorev, K. J. Kim, S. Chattopadhyay, and C. V. Shank, X-Ray Based Subpicosecond Electron Bunch Characterization Using 90 ${ }^{\circ}$ Thomson Scattering, Phys. Rev. Lett. 77, 4182 (1996). 
[4] W. S. Graves et al., Compact x-ray source based on burstmode inverse Compton scattering at $100 \mathrm{kHz}$, Phys. Rev. ST Accel. Beams 17, 120701 (2014).

[5] F. E. Carroll, M. H. Mendenhall, R. H. Traeger, C. Brau, and J. W. Waters, Pulsed tunable monochromatic X-ray beams from a compact source: New opportunities, Am. J. Roentgenol. 181, 1197 (2003).

[6] P. Oliva, M. Carpinelli, B. Golosio, P. Delogu, M. Endrizzi, J. Park, I. Pogorelsky, V. Yakimenko, O. Williams, and J. Rosenzweig, Quantitative evaluation of single-shot inline phase contrast imaging using an inverse compton x-ray source, Appl. Phys. Lett. 97, 134104 (2010).

[7] G. H. Choi et al. Photon activated therapy (PAT) using monochromatic Synchrotron X-rays and iron oxide nanoparticles in a mouse tumor model: Feasibility study of PAT for the treatment of superficial malignancy, Radiat. Oncol. 7, 184 (2012).

[8] D. J. Gibson et al., A picosecond Compton scattering x-ray source for advanced backlighting and time-resolved material studies, Phys. Plasmas 11, 2857 (2004).

[9] F. H. O'Shea, O. Williams, G. Andonian, S. Barber, Y. Sakai, J. B. Rosenzweig, I. Pogorelsky, M. Fedurin, K. Kusche, and V. Yakimenko, Single shot diffraction of picosecond 8.7-keV x-ray pulses, Phys. Rev. ST Accel. Beams 15, 020702 (2012).

[10] V. Petrillo, A. Bacci, C. Curatolo, I. Drebot, A. Giribono, C. Maroli, A. R. Rossi, L. Serafini, P. Tomassini, C. Vaccarezza, and A. Variola, Polarization of X-gamma radiation produced by a Thomson and Compton inverse scattering, Phys. Rev. ST Accel. Beams 18, 110701 (2015).

[11] F. V. Hartemann, W. J. Brown, D. J. Gibson, S. G. Anderson, A. M. Tremaine, P. T. Springer, A. J. Wootton, E. P. Hartouni, and C. P. J. Barty, High-energy scaling of Compton scattering light sources, Phys. Rev. ST Accel. Beams 8, 100702 (2005).

[12] J. Medalia, CRS Report for Congress, Congressional Research Service No. 7-5700 R40154 (2010), pp. 12-13, https://fas.org/sgp/crs/nuke/R40154.pdf.

[13] T. W. B. Kibble, Frequency shift in high-intensity compton scattering, Phys. Rev. 138, B740 (1965).

[14] J. B. Rosenzweig and O. Williams, Limits on production of narrow band photons from inverse Compton scattering, Int. J. Mod. Phys. A 22, 4333 (2007).

[15] G. A. Krafft, Spectral Distributions of Thomson-Scattered Photons from High-Intensity Pulsed Lasers, Phys. Rev. Lett. 92, 204802 (2004).

[16] C. A. Brau, Oscillations in the spectrum of nonlinear Thomson-backscattered radiation, Phys. Rev. ST Accel. Beams 7, 020701 (2004).

[17] G. A. Krafft, A. Doyuran, and J. B. Rosenzweig, Pulsedlaser nonlinear Thomson scattering for general scattering geometries, Phys. Rev. E 72, 056502 (2005).

[18] T. Heinzl, D. Seipt, and B. Kämpfer, Kämpfer, Beam-shape effects in nonlinear Compton and Thomson scattering, Phys. Rev. A 81, 022125 (2010).
[19] B. Terzić, K. Deitrick, A. S. Hofler, and G. A. Krafft, Narrow-Band Emission in Thomson Sources Operating in the High-Field Regime, Phys. Rev. Lett. 112, 074801 (2014).

[20] B. Terzić, Cody Reeves, and Geoffrey A. Krafft, Combining harmonic generation and laser chirping to achieve high spectral density in Compton sources, Phys. Rev. Accel. Beams 19, 044403 (2016).

[21] V. Petrillo, G. Dattoli, I. Drebot, and F. Nguyen, Compton Scattered X-Gamma Rays with Orbital Momentum, Phys. Rev. Lett. 117, 123903 (2016).

[22] Y. Sakai et al., Observation of redshifting and harmonic radiation in inverse Compton scattering, Phys. Rev. ST Accel. Beams 18, 060702 (2015).

[23] M. Babzien et al., Observation of the Second Harmonic in Thomson Scattering from Relativistic Electrons, Phys. Rev. Lett. 96, 054802 (2006).

[24] O. Williams et al., Characterization results of the BNL ATF Compton X-ray source using K-edge absorbing foils, Nucl. Instrum. Methods 608, S18 (2009).

[25] Y. Kamiya et al., X-ray spectrometer for observation of nonlinear compton scattering, Joint 28th ICFA Advanced Beam Dynamics and Advanced \& Novel Accelerators Workshop: Quantum Aspects of Beam Physics, edited by Pisin Chen and Kevin Reil (World Scientific, Singapore, 2004), p. 103.

[26] M. N. Polyanskiy, I. V. Pogorelsky, and V. Yakimenko, Picosecond pulse amplification in isotopic $\mathrm{CO}_{2}$ active medium, Opt. Express 19, 7717 (2011).

[27] P. B. Corkum, A. J. Alcock, and K. E. Leopold, Electron beam controlled transmission of $10 \mu \mathrm{m}$ radiation in semiconductors, J. Appl. Phys. 50, 3079 (1979).

[28] S. G. Rykovanov, C. G. R. Geddes, C. B. Schroeder, E. Esarey, and W. P. Leemans, Controlling the spectral shape of nonlinear Thomson scattering with proper laser chirping, Phys. Rev. Accel. Beams 19, 030701 (2016).

[29] G. A. Krafft, E. Johnson, K. Deitrick, B. Terzić, R. Kelmar, T. Hodges, W. Melnitchouk, and J. R. Delayen, Laser pulsing in linear Compton scattering, Phys. Rev. Accel. Beams 19, 121302 (2016).

[30] B. Terzić and G. A. Krafft, Controlling the spectral shape of nonlinear Thomson scattering with proper laser chirping, Phys. Rev. Accel. Beams 19, 098001 (2016).

[31] B. H. Failor, S. Wong, J. C. Riordan, L. T. Hudson, C. M. O'Brien, S. M. Seltzer, S. Seiler, L. Pressley, and D. Y. Lojewski, Bent-crystal Laue spectrograph for measuring X-ray spectra $(15<E<100 \mathrm{KeV})$, Rev. Sci. Instrum. 77, 10 F314 (2006).

[32] Michael J. Haugh and Richard Stewart, Measuring curved crystal performance for a high-resolution, imaging x-ray spectrometer, X-Ray Opt. Instrum. 2010, 583626 (2010).

[33] S. G. Podorov and E. Förster, Theory of x-ray diffraction on asymmetrically cut and bent crystals, Theory of x-ray diffraction on asymmetrically cut and bent crystals, Phys. Status Solidi A 220, 829 (2000). 